\title{
L'utilisation d'un bandage camoufle une lésion traumatique du bassin chez un homme de 29 ans
}

\author{
K.T. Matthew Seah MRCSEd, James W. MacKay PhD
}

Citation : CMAJ 2021 February 8;193:E216. doi : 10.1503/cmaj.201114-f

Voir la version anglaise de l'article ici : www.cmaj.ca/lookup/doi/10.1503/cmaj.201114

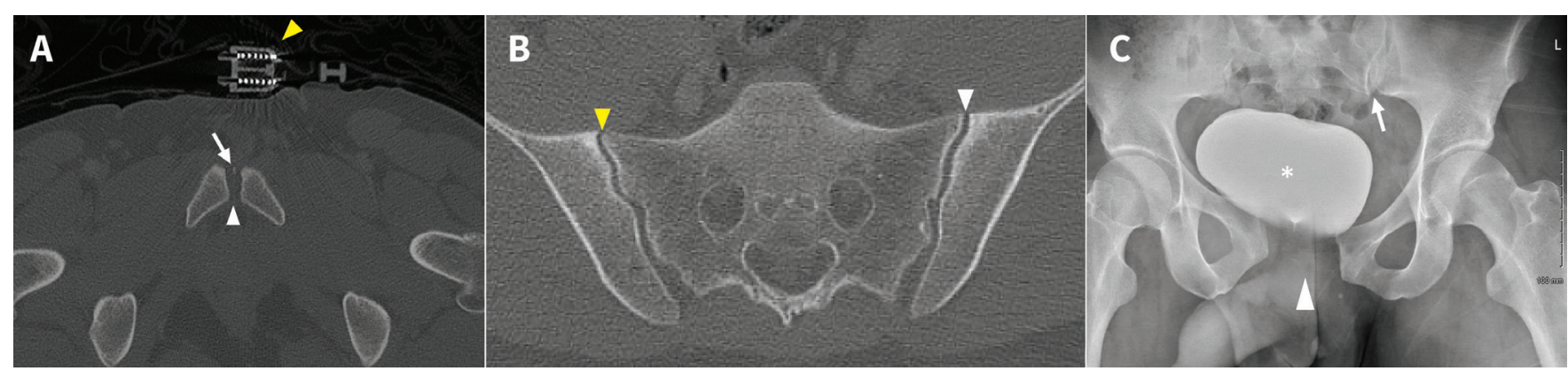

Figure 1: A) Cliché de tomodensitométrie (TDM) axiale (fenêtres osseuses) de la symphyse pubienne d'un homme de 29 ans, montrant une symphyse pubienne apparemment congruente (pointe de flèche blanche) et le bandage pelvien (pointe de flèche jaune). Notons la présence d'un petit fragment osseux dans l'espace articulaire de la symphyse pubienne (flèche blanche), possiblement le résultat d'une fracture par arrachement (non détecté à la lecture initiale). B) Cliché de TDM axiale (fenêtres osseuses) des articulations sacro-iliaques montrant des articulations congruentes, sans fracture. Avec du recul, l'articulation sacro-iliaque gauche (pointe de flèche blanche) est possiblement plus large que la droite (pointe de flèche jaune), mais la différence est extrêmement subtile. C) Cliché de radiographie antéro-postérieure obtenu après le retrait du bandage pelvien, montrant un diastasis de la symphyse pubienne (pointe de flèche blanche) et un élargissement subtil de l'articulation sacro-iliaque gauche (flèche blanche). La vessie (astérisque), remplie d'un produit de contraste à base d'iode pour la TDM, est poussée vers la droite par l'hématome pelvien observé par TDM (annexe 1, accessible en anglais au www.cmaj.ca/lookup/doi/10.1503/cmaj.201114/tab-related-content).

$\mathbf{U}$ n motocycliste de 29 ans est transporté dans un centre de traumatologie après une collision avec un garde-fou sur une autoroute. Comme le patient présentait une instabilité hémodynamique, l'équipe de soins préhospitaliers a appliqué un bandage pelvien. Avant son arrivée à l'hôpital, le patient avait une fréquence cardiaque de 144 battements/ minute, une fréquence respiratoire de 28 respirations/minute et une pression artérielle de 100/65 mm Hg. Au service des urgences, le patient est alerte et dit ressentir une douleur tolérable à l'abdomen inférieur. À l'examen, on constate un abdomen souple et des fractures ouvertes aux membres inférieurs avec lésion vasculaire soupçonnée. Une tomodensitométrie (TDM) d'urgence ne révèle aucune lésion intra-abdominale ni fracture du bassin, mais on y voit un hématome pelvien sans signe de saignement actif (annexe 1, accessible en anglais au www.cmaj.ca/lookup/ doi/10.1503/cmaj.201114/tab-related-content).

Après la réanimation initiale, le patient est stable sur le plan hémodynamique, et le bandage pelvien est retiré. Une radiographie pelvienne effectuée après le retrait du bandage (conformément aux directives locales) révèle un diastasis de la symphyse pubienne et une lésion à l'articulation sacro-iliaque gauche (figure 1A). Un examen rétrospectif du tomodensitogramme (bandage encore en place) permet d'observer quelques signes indiquant une lésion plus grave que ce qui avait été initialement conclu : hématome pelvien, petite fracture par arrachement à la symphyse pubienne (figure 1B) et élargissement subtil de l'articulation sacro-iliaque gauche (figure 1C) ${ }^{1}$.

Les directives préhospitalières locales préconisent l'application d'un bandage si on soupçonne une lésion pelvienne associée à un traumatisme contondant à haute énergie avec instabilité hémodynamique (ou pression systolique $<110 \mathrm{~mm} \mathrm{Hg}$ ) 2 . Il est possible de ne pas détecter une lésion grave au bassin en présence d'un bandage bien appliqué ${ }^{3}$, particulièrement dans le cas des lésions purement ligamentaires découlant d'une compression antéropostérieure. Les centres de traumatologie devraient aussi se doter d'un protocole clair de retrait des bandages ${ }^{4}$.

Les praticiens devraient garder en tête qu'un bandage pelvien bien appliqué peut camoufler des lésions ligamentaires 
associées à une fracture du bassin de type « livre ouvert »; il faudrait donc systématiquement procéder à une radiographie classique du bassin après le retrait du bandage pelvien chez les patients en traumatologie.

\section{Références}

1. Dreizin D, Nascone J, Davis DL, et al. Can MDCT unmask instability in binderstabilized pelvic ring disruptions? AJR Am J Roentgenol 2016;207:1244-51.

2. Scott I, Porter K, Laird C, et al. The prehospital management of pelvic fractures: initial consensus statement. Emerg Med J 2013;30:1070-2.

3. Clements J, Jeavons $\mathrm{R}$, White $\mathrm{C}$, et al. The concealment of significant pelvic injuries on computed tomography evaluation by pelvic compression devices. $J$ Emerg Med 2015;49:675-8.

4. British Orthopaedic Association audit standards for trauma: the management of patients with pelvic fractures. London (UK): British Orthopaedic Association (BOA); 2018

Intérêts concurrents : James MacKay déclare avoir reçu du soutien à la recherche et des honoraires de consultation de GlaxoSmithKline et de GE Healthcare. Aucun autre intérêt concurrent n'a été déclaré.

Cet article a été révisé par des pairs.

Les auteurs ont obtenu le consentement du patient.

Affiliations : Département d'orthopédie (Seah), Université Cambridge, Hôpital Addenbrooke, Cambridge, R.-U.; Département de radiologie (MacKay), Faculté de médecine Norwich, Université d'East Anglia, Norwich, R.-U.

Propriété intellectuelle du contenu : Il s'agit d'un article en libre accès distribué conformément aux modalités de la licence Creative Commons Attributions (CC BY-NC-ND 4.0), qui permet l'utilisation, la diffusion et la reproduction dans tout médium à la condition que la publication originale soit adéquatement citée, que l'utilisation se fasse à des fins non commerciales (c.-à-d. recherche ou formation) et qu'aucune modification ni adaptation n'y soit apportée. Voir : https://creativecommons.org/licenses/by-nc-nd/4.0/deed.fr.

Correspondance : K. Seah, matt.seah@doctors.org.uk

Les images cliniques sont choisies pour leur caractère particulièrement intéressant, classique ou impressionnant. Toute soumission d'image de haute résolution claire et bien identifiée doit être accompagnée d'une légende aux fins de publication. On demande aussi une brève explication (300 mots maximum) de la portée éducative des images, et des références minimales. Le consentement écrit du patient au regard de la publication doit être obtenu avant la soumission. 